\title{
LETTER
}

Acute myeloid leukemia

\section{Single base substitution mutational signatures in pediatric acute myeloid leukemia based on whole genome sequencing}

\author{
Rebeqa Gunnarsson $\mathbb{1}^{1} \cdot$ Minjun Yang $\mathbb{1}^{1} \cdot$ - Linda Olsson-Arvidsson ${ }^{1,2} \cdot$ Andrea Biloglav $^{1} \cdot$ Mikael Behrendtz $^{3}$. \\ Anders Castor ${ }^{4}$ - Kajsa Paulsson ID $^{1}$ - Bertil Johansson ${ }^{1,2}$
}

Received: 5 October 2020 / Revised: 9 March 2021 / Accepted: 23 March 2021 / Published online: 16 April 2021

(c) The Author(s) 2021. This article is published with open access

\section{To the Editor:}

Whole genome sequencing (WGS) of neoplasms not only offers a comprehensive genome-wide detection of gene fusions/other structural variants (SVs), gene mutations, and copy number abnormalities (CNAs) but also provides information on acquired mutational profiles, including localized hypermutations (kataegis) and single base substitution (SBS) mutational signatures. The latter are recurring trinucleotide patterns of somatic single nucleotide variants (SNVs) and their flanking nucleotides that are, in some instances, associated with the etiology or pathogenesis of neoplastic disorders, for example $\mathrm{C}>\mathrm{T}$ transitions in $\mathrm{UV}$ associated melanoma, $\mathrm{C}>\mathrm{A}$ transversions in smokinginduced lung cancer, and $\mathrm{C}>\mathrm{T}$ transitions and $\mathrm{C}>\mathrm{G}$ transversions caused by overactive APOBEC enzymes [1, 2]. In addition, WGS allows detection of variants in non-coding regulatory elements (REs), such as enhancers and promoters, resulting in de-regulated gene transcription [3]. However, the few WGS studies of pediatric acute myeloid leukemia (AML) reported to date have not ascertained SBS

These authors contributed equally: Rebeqa Gunnarsson, Minjun Yang

Supplementary information The online version contains supplementary material available at https://doi.org/10.1038/s41375021-01242-0.

Rebeqa Gunnarsson

rebeqa.gunnarsson@med.lu.se

1 Division of Clinical Genetics, Department of Laboratory Medicine, Lund University, Lund, Sweden

2 Department of Clinical Genetics and Pathology, Division of Laboratory Medicine, Lund, Sweden

3 Department of Pediatrics, Linköping University Hospital, Linköping, Sweden

4 Department of Pediatrics, Skåne University Hospital, Lund, Sweden mutational signatures or variants in REs [4-6]. We performed WGS on 20 pediatric AML cases (Supplementary Information and Supplementary Fig. S1), focusing on SBS profiles, variants in REs, and novel gene fusions and mutations.

Apart from confirming all gene fusions detected in clinical routine, the SV analysis revealed three novel inframe fusions: PLEKHA5-ADAMTS20, RAB11FIP2NEURL4, and TCF3-HOXB9 (Supplementary Information, Supplementary Tables S1 and S2, and Supplementary Figs. S2 and S3). The TCF3-HOXB9 fusion recombines two transcription factor genes that are important for differentiation, such as lymphopoiesis, and proliferation and that are expressed in bone marrow (BM) and lymph nodes (LNs) (Supplementary Table S2). In addition, TCF3 is a partner in several other fusion genes in, e.g., acute lymphoblastic leukemia (ALL). The PLEKHA5-ADAMTS2O fusion involves two genes that are often fused to other genes in, mainly, epithelial malignancies but that are also normally expressed in BM and LNs. (Supplementary Table S2). RAB11FIP2-NEURL4 rearranges RAB11FIP2, expressed in $\mathrm{BM}$ and LNs, with the mitotic gene NEURLA, which is fused to, for example, MSI2 in malignant melanoma (supplementary Table 2). An XPO1-TNRC18 fusion, previously reported in a single case of ALL [7], was also identified. In total, $13(65 \%)$ of the 20 AMLs harbored WGS-identified fusion genes (Supplementary Table S1). Although we cannot exclude the possibility that the novel fusions were merely byproducts of the genetic instability present in many tumor types-such "passenger fusions" are commonly identified by various types of massively parallel sequencing [8]-we consider this unlikely. First, all gene partners in the novel fusions, except $R A B 11 F I P 2$, have previously been reported to be rearranged with other genes in human malignancies (Supplementary Table S2; https://mitelmanda tabase.isb-cgc.org/); this increases the likelihood that they are pathogenetically important. Second, the fact that the fusion genes were identified by WGS clearly shows that 
they existed on the DNA level and, hence, were not transcription-induced $[8,9]$.

A total of 34 CNAs (24 losses and 10 gains; median 1.5 CNAs/case) and two uniparental isodisomies were detected; none of the cases displayed chromothripsis (Supplementary Information and Supplementary Table S3). The frequencies of imbalances did not differ between cases with or without (w/wo) fusion genes $(P=0.3)$. Of the 10920 somatic variants identified (Supplementary Table S1), 10,492 (96\%) were SNVs and 428 (4\%) small insertions/deletions (indels). There were between 73 and $1198 \mathrm{SNVs} /$ indels per case (median 502/case), corresponding to 0.024-0.386 $\mathrm{SNV}$ s/indels per Mb. This low frequency of SNVs/indels agrees well with a previous study showing that pediatric AMLs harbor fewer mutations than other childhood cancers [10]. Furthermore, the rainfall plot analyses revealed kataegic regions in only two cases (Supplementary Fig. S4). The most common type of SNV was a $\mathrm{C}>\mathrm{T}$ transition (48\%), followed by $\mathrm{C}>\mathrm{A}$ transversions $(17 \%), \mathrm{T}>\mathrm{C}$ transitions (15\%), $\mathrm{T}>\mathrm{G}(7 \%), \mathrm{T}>\mathrm{A}(7 \%)$, and $\mathrm{C}>\mathrm{G}(6 \%)$ transversions (Fig. 1). All transitions and transversions, except $\mathrm{C}>\mathrm{A}$, increased significantly with age (Supplementary Fig. S5), as expected considering that many acquired SNVs are known to occur in a clockwise manner [11]. Neither the total mutational burden nor the different types of substitution differed significantly between cases w/wo fusion genes (data not shown).

The SBS mutational signature analysis revealed that signature 1 (SBS1) was the most common one, detected in all 20 AMLs with relative contributions of $15-34 \%$ per case (median 25\%; Fig. 1). It is associated with a predominance of $\mathrm{C}>\mathrm{T}$ transitions resulting from endogenous, spontaneous deamination of 5-methylcytosines and increasing by age [1]. The second most frequent signature was SBS18, found in 15 of the AMLs (median relative contribution of $8.2 \%$ ), which is characterized by $\mathrm{C}>\mathrm{A}$ transversions and possibly associated with damage caused by reactive oxygen species (ROS) (https://cancer.sanger.ac.uk/cosmic/signatures/SBS/ SBS18.tt). The SBS39, SBS37, and SBS32 signatures were detected in 18,15 , and 13 of the cases, with median values of relative contributions of $4.0 \%, 3.9 \%$, and $3.2 \%$, respectively. The etiologies of SBS37 and SBS39 are unknown, whereas SBS32 has been associated with prior treatment with azathioprine (https://cancer.sanger.ac.uk/ cosmic/signatures/SBS). In the hierarchical cluster analysis of the above-mentioned signatures, the RUNXI-RUNXT1positive cases clustered together in a separate branch, most likely because of the high proportions of SBS18 signatures/ $\mathrm{C}>\mathrm{A}$ transversions in these cases (Supplementary Fig. S6). Interestingly, frequent $\mathrm{C}>\mathrm{A}$ transversions in RUNXIRUNXT1-positive cases have previously been reported [4] and, although SBS mutational profiling was not performed in that study, it supports the association between $\mathrm{C}>\mathrm{A}$ transversions, SBS18, and $\mathrm{t}(8 ; 21)$ AML identified herein. In order to validate the association between RUNX1RUNX1T1, C $>$ A transversions, and SBS18, we utilized the TCGA dataset [12]; however, it should be stressed that this is based on whole exome sequencing data and hence is much less informative than WGS data with regard to number of SNVs. First, we compared the frequencies of the different SBS types in RUNX1-RUNXIT1-positive and -negative cases in our cohort and in the TCGA dataset. As seen in Supplementary Table S4, a relatively high frequency of $\mathrm{C}>\mathrm{A}$ transversions was observed in the RUNX1RUNXT1-positive cases also in the TCGA cohort. Furthermore, the SBS18 signature was observed in the RUNX1RUNXIT1-positive group in the TCGA dataset; in contrast, no SBS18 signature was identified in the RUNX1RUNXIT1-negative subgroups (Supplementary Table S5). Although based on whole exome sequencing data, the TCGA cohort thus provides some support for the association between $\mathrm{C}>\mathrm{A}$ transversions, SBS18, and $\mathrm{t}(8 ; 21)$ in AML.

Comparing the SNVs/indels found at diagnosis and relapse of cases 1 and 2 revealed that 51\% (case 1) and 33\% (case 2) of the SNVs/indels at relapse were identical to those detected at diagnosis; the other SNVs/indels were either novel or lost at relapse (Supplementary Fig. S7). Interestingly, the SBS mutational signatures were similar at diagnosis and relapse (data no shown), showing that the induction and consolidation therapies did not result in different signatures at relapse.

Of the 10,920 SNVs/indels, $123(1.1 \%)$ occurred within coding genes (Supplementary Table S6) and 110 of these could be confirmed by deep sequencing, whole exome sequencing, or Sanger sequencing. Eighty-nine of the verified SNVs/indels in 84 different genes were considered pathogenic, either by default by being truncating or by being classified as such by SIFT and/or PolyPhen. Gene ontology data on molecular functions were available for 43 of the 84 genes: the most frequent functions were transcriptional regulation (67\%) and metal ion binding (67\%) (Fig. 2 and Supplementary Fig. S8). Apart from genes previously reported to be mutated in pediatric AML, e.g., DNMT3A, GATA2, JAK3, NCOR1, and NOTCH1 [4, 13], we identified pathogenic SNVs/indels in 33 genes (Supplementary Table S6) previously not implicated in AML, such as RASL11A (RAS signaling), ATPB5 (mitochondrial metabolism), and ASCC3, MACF1, USF2, ZFAT, and ZNF251 (transcriptional regulation).

The analysis of the 10,920 somatic SNVs/indels revealed that $133(1.2 \%)$ of them occurred in REs, mainly enhancers $(41 \%)$ and promoters (26\%) (Supplementary Information and Supplementary Table S7). The potential targets of the RE variants comprised 656 targets-506 (77\%) genes and $150(23 \%)$ ncRNAs. Only six genes and one ncRNA were 

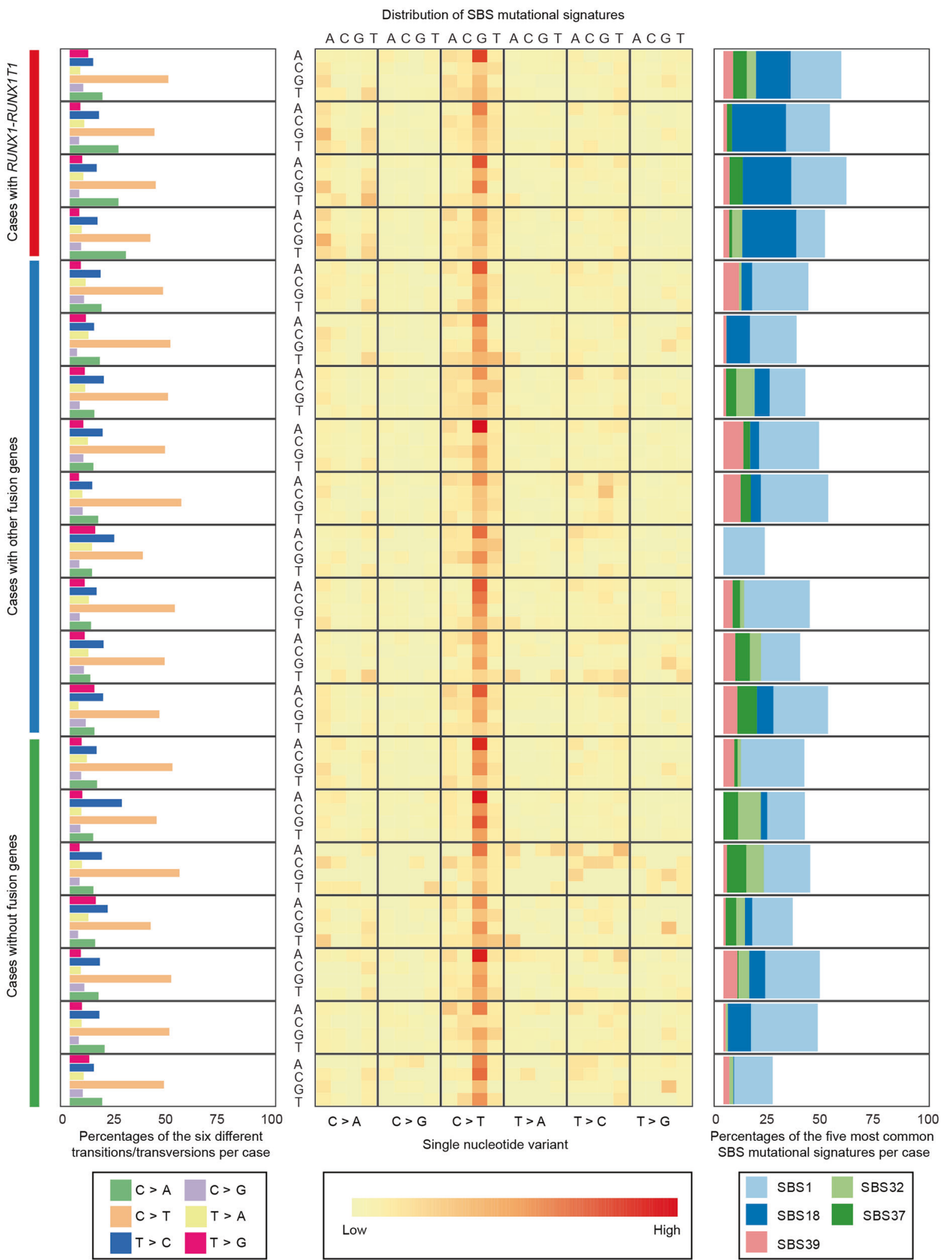

Fig. 1 Overview of the transition and transversion types and the single base substitution (SBS) mutational signatures in the pediatric acute myeloid leukemias cases. Left panel: the distribution of the six different transition and transversion types per case. Middle panel: heat map showing the distribution of the SBS signatures in each case. Right panel: The frequencies of the five overall most common signatures (SBS1, SBS18, SBS32, SBS37, and SBS39) per case. 


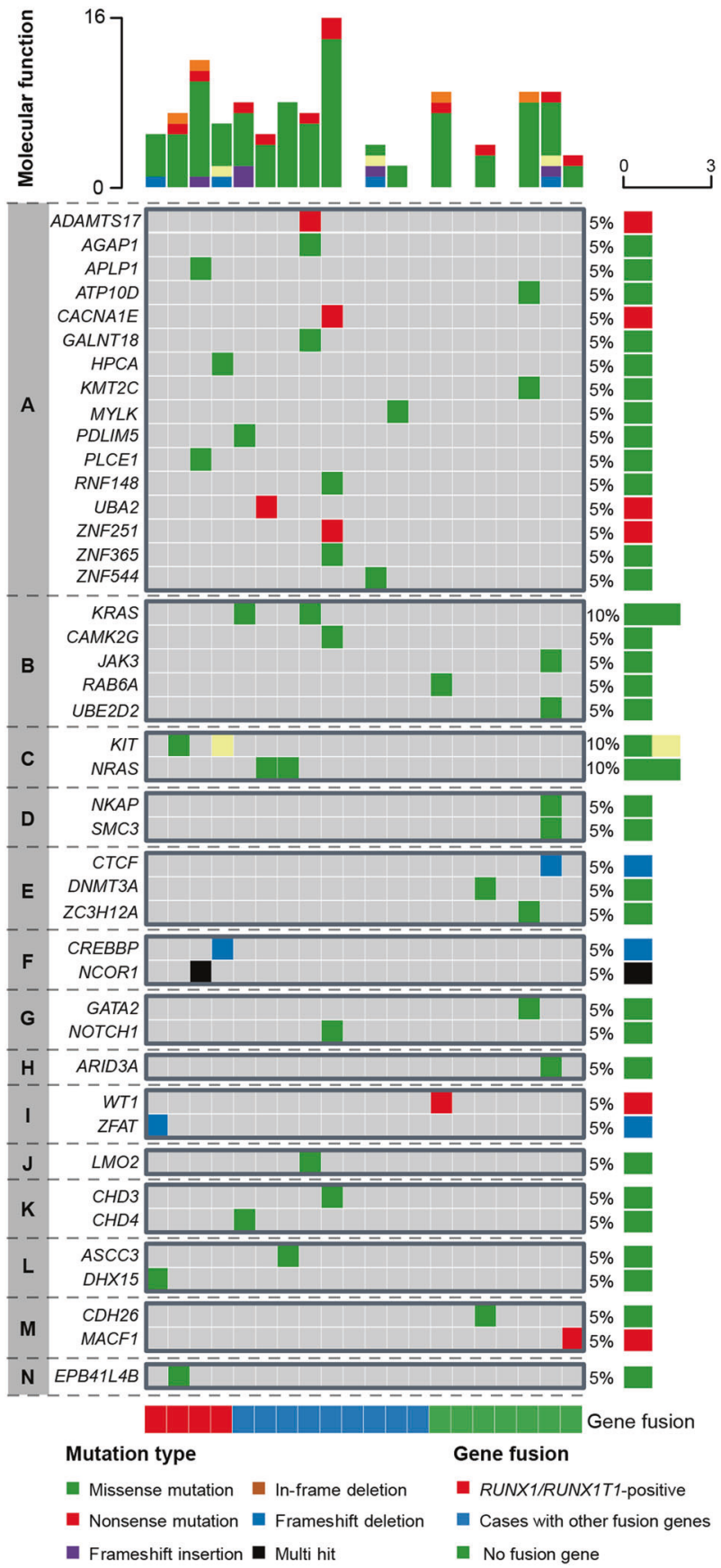

Fig. 2 Overview of gene ontology (GO)-annotated molecular functions of the 43 genes with pathogenic variants. $A-N$ indicate different GO functions: A metal ion binding; $\mathbf{B}$ nucleotide binding; $\mathbf{C}$ metal ion and nucleotide binding; $\mathbf{D}$ chromatin binding; $\mathbf{E}$ metal ion and chromatin binding; $\mathbf{F}$ chromatin and polymerase II activating transcription factor binding; G metal ion, chromatin/DNA binding, and transcription activator; $\mathbf{H}$ chromatin/DNA binding and transcription activator; I metal ion, DNA binding, and transcription activator; $\mathbf{J}$ metal ion, DNA and RNA polymerase II activating transcription factor binding, and transcription activator; $\mathbf{K}$ metal ion binding and helicase activity; $\mathbf{L}$ helicase activity; $\mathbf{M}$ metal ion binding and cytoskeletal protein binding; $\mathbf{N}$ cytoskeletal protein binding. recurrently targeted (Supplementary Table S7), suggesting that the RE variants did not affect specific genes/pathways.

In conclusion, the present WGS analysis of pediatric AML identified both known and novel fusion genes (PLEKHA5-ADAMTS20, RAB11FIP2-NEURL4, and TCF3HOXB9) and revealed relatively few CNAs and SNAs/ indels as compared with other malignancies. Furthermore, regions with kataegis were rare and there were no signs of chromothripsis. Thus, childhood AML is characterized by a low degree of genomic complexity and mutational burden. Of the acquired variants detected, only a minority targeted REs $(\sim 1 \%)$ and coding genes $(\sim 1 \%)$. However, a large proportion $(39 \%)$ of the coding genes had previously not been implicated in AML. Although fusion genes are considered strong driver mutations, requiring few additional hits for generating overt leukemia [14], this was not reflected by differences in CNAs, SNVs/indels, overall genomic complexity, functions of the mutated genes, or SBS mutational signatures between fusion-positive and -negative cases. However, RUNX1-RUNXT1-positive cases were for the first time associated with a higher prevalence of the ROS-associated SBS18 signature, likely due to the high frequency of $\mathrm{C}>\mathrm{A}$ transversions in this AML subtype. Although the correlation between SBS18 and $t(8 ; 21)$ should be considered preliminary-additional studies are needed to confirm or refute this-the present finding indicates that DNA damage caused by ROS $[14,15]$ may be of particular importance in RUNX1-RUNXT1-positive AML.

\section{Data availability}

The dataset generated during the current study fall under the GDPR regulations for sharing of personal data and will therefore be made available in the EGA-SE depository upon its completion. Until then, the data are available from the corresponding author upon request through the following DOI: https://figshare.com/s/5a1ca3f39611c39bfaae (WGS dataset). Supplementary information is available at Leukemia's website.

Acknowledgements This study was supported by grants from the Swedish Cancer Society (20 $0792 \mathrm{PjF})$, the Swedish Childhood Cancer Foundation (PR2018-0008 and TJ2020-0024), the Swedish Research Council (2020-01164), and Governmental Funding of Clinical Research within the National Health Service. The computations were performed on resources provided by LUNARC through Lund University under Project LSENS 2018/3-4.

Author contributions RG planned and performed research and wrote the paper, MY performed the bioinformatics analysis and wrote the paper. $\mathrm{LO}, \mathrm{AB}$, and $\mathrm{KP}$ performed research. $\mathrm{MB}$ and $\mathrm{AC}$ provided patient samples and clinical data. BJ planned research and wrote the paper. The paper was reviewed and approved by all the authors. 
Funding The Swedish Cancer Society (20 $0792 \mathrm{PjF}$ ), the Swedish Childhood Cancer Foundation (PR2018-0004 and TJ2020-0024), the Swedish Research Council (2020-01164), and Governmental Funding of Clinical Research within the National Health Service.

\section{Compliance with ethical standards}

Conflict of interest The authors declare no competing interests.

Publisher's note Springer Nature remains neutral with regard to jurisdictional claims in published maps and institutional affiliations.

Open Access This article is licensed under a Creative Commons Attribution 4.0 International License, which permits use, sharing, adaptation, distribution and reproduction in any medium or format, as long as you give appropriate credit to the original author(s) and the source, provide a link to the Creative Commons license, and indicate if changes were made. The images or other third party material in this article are included in the article's Creative Commons license, unless indicated otherwise in a credit line to the material. If material is not included in the article's Creative Commons license and your intended use is not permitted by statutory regulation or exceeds the permitted use, you will need to obtain permission directly from the copyright holder. To view a copy of this license, visit http://creativecommons. org/licenses/by/4.0/.

\section{References}

1. Alexandrov LB, Nik-Zainal S, Wedge DC, Aparicio SA, Behjati $\mathrm{S}$, Biankin AV, et al. Signatures of mutational processes in human cancer. Nature. 2013;500:415-21.

2. Nakagawa H, Fujita M. Whole genome sequencing analysis for cancer genomics and precision medicine. Cancer Sci. 2018;109:513-22.

3. Fredriksson NJ, Ny L, Nilsson JA, Larsson E. Systematic analysis of noncoding somatic mutations and gene expression alterations across 14 tumor types. Nat Genet. 2014;46:1258-63.
4. Bolouri H, Farrar JE, Triche T Jr, Ries RE, Lim EL, Alonzo TA, et al. The molecular landscape of pediatric acute myeloid leukemia reveals recurrent structural alterations and age-specific mutational interactions. Nat Med. 2018;24:103-12.

5. Faber ZJ, Chen X, Gedman AL, Boggs K, Cheng J, Ma J, et al. The genomic landscape of core-binding factor acute myeloid leukemias. Nat Genet. 2016;48:1551-6.

6. Yoshida K, Toki T, Okuno Y, Kanezaki R, Shiraishi Y, SatoOtsubo A, et al. The landscape of somatic mutations in Down syndrome-related myeloid disorders. Nat Genet. 2013;45:1293-9.

7. Gu Z, Churchman M, Roberts K, Li Y, Liu Y, Harvey RC, et al. Genomic analyses identify recurrent $M E F 2 D$ fusions in acute lymphoblastic leukaemia. Nat Commun. 2016;7:13331.

8. Mertens F, Johansson B, Fioretos T, Mitelman F. The emerging complexity of gene fusions in cancer. Nat Rev Cancer. 2015;15:371-81.

9. Panagopoulos I, Thorsen J, Gorunova L, Micci F, Heim S. Sequential combination of karyotyping and RNA-sequencing in the search for cancer-specific fusion genes. Int $\mathrm{J}$ Biochem Cell Biol. 2014;53:462-5.

10. Grobner SN, Worst BC, Weischenfeldt J, Buchhalter I, Kleinheinz $\mathrm{K}$, Rudneva VA, et al. The landscape of genomic alterations across childhood cancers. Nature. 2018;555:321-7.

11. Zhang L, Dong X, Lee M, Maslov AY, Wang T, Vijg J. Singlecell whole-genome sequencing reveals the functional landscape of somatic mutations in B lymphocytes across the human lifespan. Proc Natl Acad Sci USA. 2019;116:9014-9.

12. Ley TJ, Miller C, Ding L, Raphael BJ, Mungall AJ, et al. Genomic and epigenomic landscapes of adult de novo acute myeloid leukemia. N Engl J Med. 2013;368:2059-74.

13. Tarlock K, Zhong S, He Y, Ries R, Severson E, Bailey M, et al. Distinct age-associated molecular profiles in acute myeloid leukemia defined by comprehensive clinical genomic profiling. Oncotarget. 2018;9:26417-30.

14. Hyrenius-Wittsten A, Pilheden M, Sturesson H, Hansson J, Walsh MP, Song G, et al. De novo activating mutations drive clonal evolution and enhance clonal fitness in KMT2A-rearranged leukemia. Nat Commun. 2018;9:1770.

15. Sillar JR, Germon ZP, DeIuliis GN, Dun MD. The role of reactive oxygen species in acute myeloid leukaemia. Int $\mathrm{J}$ Mol Sci. 2019;20:1-20. 